\title{
Asthma, COPD and SARS-CoV-2 infection (COVID-19): potential mechanistic insights
}

\begin{abstract}
To the Editor:
Copyright @The authors 2021

This version is distributed under the terms of the Creative Commons Attribution NonCommercial Licence 4.0. For commercial reproduction rights and permissions contact permissions@ersnet.org

Received: 29 March 2021 Accepted: 24 June 2021

\section{๑@®@}

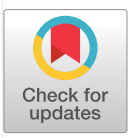

We read with interest the manuscripts [1, 2] and the accompanying editorial [3] describing the risk of severe disease and infection with severe acute respiratory syndrome coronavirus 2 (SARS-CoV-2) and asthma, but are struck by the difference seen with risk of complications from asthma and coronavirus disease 2019 (COVID-19) compared to the experience during the 2009 influenza pandemic, where people with asthma were clearly at heightened risk, at least of hospitalisation [4]. In contrast, those who smoke or have COPD, appear at greater risk. Observational studies will never be able to provide evidence of cause and effect and a greater understanding of the mechanisms of susceptibility to infection with SARS-CoV-2 is also required. Hence, we would like to take this further and enhance discussion on potential mechanisms (figure 1).

SARS-CoV-2 shows a strong affinity towards the human angiotensin-converting enzyme 2 (ACE2) receptor through their densely glycosylated spike (S) protein, which forms the initiation step for the virus attachment and subsequent entry into human cells [5]. The virus binds to the ACE2 receptor and requires the transmembrane serine protease 2 (TMPRSS2) to cleave the viral spike protein in order to enter a cell [5]. This step appears to be facilitated by endosomal proteases such as cathepsin-L (CTL) and enhanced by the Furin protein, while ADAM-17 may promote uptake of SARS-CoV-2 [6].

In asthma, we found reduced expression of ACE2 in the lower airways of people with asthma, both in terms of protein and gene expression [7]. We also found in asthma that Furin expression was lower and ADAM-17 expression elevated. This provides a potential mechanism by which chronic type 2 airway inflammation through the effect of interleukin (IL)-13 on the airway epithelium could increase ADAM-17, resulting in shedding and downregulation of ACE2 expression prior to infection. An important and unanswered question is whether different asthma phenotypes may harbour different risks or whether through control of type 2 airway inflammation, such as with the use of biologics, that ACE2 expression is increased and any advantage seen in asthma is then lost. This may in part explain the increased risk seen in those with more severe asthma or using biologics.

In contrast to asthma, the toxic irritants resulting from tobacco smoking and, more recently, vaping may enhance the risk of developing more severe COVID-19. Our recently published study identified that both smokers and COPD patients had upregulated expression of ACE2 on small airway epithelium, type II pneumocytes and alveolar macrophages [8]. We further reported a concomitant rise in endocytic vacuoles, including early and late endosomes EEA1, RAB-7 and lysosomal associated membrane protein-1 (LAMP-1) in the same tissue areas where ACE2 was expressed [8]. We also identified increased expression of CTL, an endo-lysosomal protease that facilitates viral S protein membrane fusion in a low $\mathrm{pH}$ environment, usually found during increased lysosomal activity. Such mechanisms could also be active in patients with asthma, but this requires further investigation. When we compared endobronchial biopsies from patients with COPD to asthmatics there was reduced ACE2 levels in the bronchial epithelium of patients with asthma [7]. It is quite possible that low levels of ACE2 in asthmatics could be due to inhaled corticosteroid use and lack of smoking in these patients. The study by IzQUIERDo et al. [2] also suggested that inhaled corticosteroid may be associated with a protective effect against SARS-CoV-2 infection.

The role of lung inflammation is critical to the viral infection and cytokine release syndrome is a significant morbidity associated with COVID-19 patients. The increase in IL-6 has been particularly called into question.

Shareable abstract (@ERSpublications)

Patients with asthma are somewhat protected from COVID-19 complications compared to COPD. This may be due to variability in viral attachment protein, ICS use, smoking/vaping status, a skewed inflammatory pattern, and dysregulated endocytic machinery. https://bit.ly/3qOcyNb

Cite this article as: Wark PAB, Pathinayake PS, Eapen MS, et al. Asthma, COPD and SARS-CoV-2 infection (COVID-19): potential mechanistic insights. Eur Respir J 2021; 58: 2100920 [DOI: 10.1183/ 13993003.00920-2021].
\end{abstract}




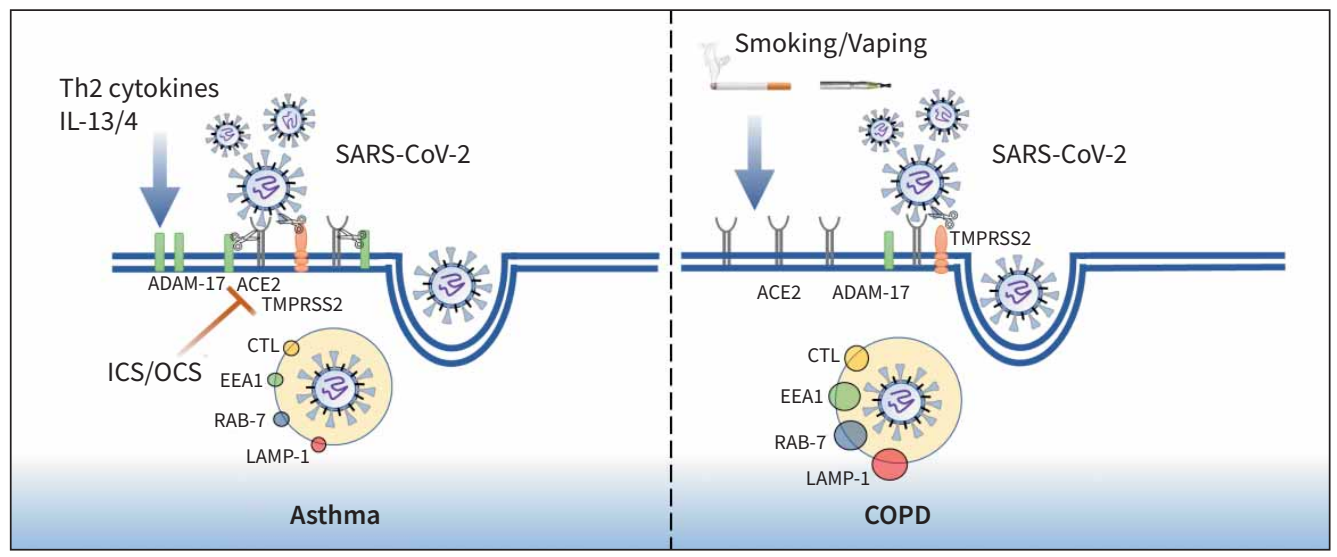

FIGURE 1 Cellular mechanisms of severe acute respiratory syndrome coronavirus 2 (SARS-CoV-2) infection in asthma and COPD. Smoking and vaping exposure upregulates angiotensin-converting enzyme 2 (ACE2) receptor expression in airway epithelium while inhaled corticosteroids (ICS) and oral corticosteroids (OCS) reduce ACE2 expression in asthma compared to COPD. Once SARS-CoV-2 binds to the ACE2 receptor via the spike protein, serine protease TMPRSS2 cleaves the viral spike protein, facilitating viral uptake and internalisation by endocytosis. The endosome- and lysosome-associated proteins EEA1, RAB-7, LAMP-1 and CTL, which facilitate viral replication within the host cell, are abundant in COPD epithelium. Th2 cytokines (IL-13) upregulate ADAM-17 expression in asthma epithelium, resulting in ACE2 shedding prior to infection.

In our previous observations, the bronchoalveolar lavage from patients with COPD had increased levels of Th2/M2 cytokines such as IL-6, CCL22, IL-4, IL-13 and IL-10, while Th1/M1 cytokines IL-12 and interferon- $\gamma$ were suppressed [9]. Similar to asthma, as mentioned above, IL-13 levels also increase in COPD, but the literature do not report any protective effects of that on ACE2 levels, if that works through ADAM-17. However, ADAM-17 deficiency has been suggested to protect against emphysema [10]. Compared to asthmatics, these observations indicate smokers and COPD patients are primed for SARS-CoV-2 infection with favourable infectious cytokine profiles, which may respond in different ways given the disease in question.

Taken together we believe that patients with asthma are somewhat protected against SARS-CoV-2 infection, which may partly be due to decrease in ACE2 levels as we reported. This could be because of type 2 inflammation or a protective effect from low to medium dose inhaled or oral corticosteroid. At the same time, asthma patients do seem to be slightly more at risk of developing COVID-19 as indicated by studies from CHOI et al. [1] and IzQuierDo et al. [2] but this may be confounded by factors of age and sex as shown in our asthma study [7]. Compared to asthma patients, smokers and those with COPD certainly have higher levels of ACE2. The underlying inflammatory cytokine profile in lung conditions is quite important to understand as that may dictate the pathogenesis of disease or be protective. It will be highly beneficial and worth exploring mechanisms which allow patients such as those with asthma to stay relatively protected against COVID-19 and how environmental factors, older age and other comorbidities further exaggerate such insidious infections. As rightly mentioned by EGER and BEL [3] in the accompanying editorial, it is hard to draw any firm conclusions from both the studies, as several factors may influence the reported incidences. Hence, understanding the biological mechanisms behind such observations will have important public health benefits, not only for COVID-19, but also for any future pandemics. COVID-19 is a dress rehearsal for the next pandemic and the one after.

Peter A.B. Wark $\oplus^{1,2}$, Prabuddha S. Pathinayake $\oplus^{1}$, Mathew Suji Eapen $\oplus^{3}$ and Sukhwinder Singh Sohal ${ }^{3}$

${ }^{1}$ Priority Research Centre for Healthy Lungs, Hunter Medical Research Institute and School of Medicine and Public Health, University of Newcastle, Newcastle, Australia. ${ }^{2}$ Dept of Respiratory and Sleep Medicine, John Hunter Hospital, Newcastle, Australia. ${ }^{3}$ Respiratory Translational Research Group, Dept of Laboratory Medicine, School of Health Sciences, College of Health and Medicine, University of Tasmania, Launceston, Australia. 
Conflict of interest: P.A.B. Wark has nothing to disclose. P.S. Pathinayake has nothing to disclose. M.S. Eapen has nothing to disclose. S.S. Sohal reports personal fees for lectures from Chiesi, outside the submitted work.

Support statement: This work was supported by Clifford Craig Foundation Launceston General Hospital. Funding information for this article has been deposited with the Crossref Funder Registry.

\section{References}

1 Choi YJ, Park JY, Lee HS, et al. Effect of asthma and asthma medication on the prognosis of patients with COVID-19. Eur Respir J 2021; 57: 2002226.

2 Izquierdo JL, Almonacid C, González Y, et al. The impact of COVID-19 on patients with asthma. Eur Respir J 2021; 57: 2003142.

3 Eger K, Bel EH. Asthma and COVID-19: do we finally have answers? Eur Respir J 2021; 57: 2004451.

4 Jha A, Dunning J, Tunstall T, et al. Patterns of systemic and local inflammation in patients with asthma hospitalised with influenza. Eur Respir J 2019; 54: 1900949.

5 Asrani P, Hasan GM, Sohal SS, et al. Molecular basis of pathogenesis of coronaviruses: a comparative genomics approach to planetary health to prevent zoonotic outbreaks in the 21st century. OMICS 2020; 24: 634-644.

6 Huang IC, Bosch BJ, Li F, et al. SARS coronavirus, but not human coronavirus NL63, utilizes cathepsin L to infect ACE2-expressing cells. J Biol Chem 2006; 281: 3198-3203.

7 Wark PAB, Pathinayake PS, Kaiko G, et al. ACE2 expression is elevated in airway epithelial cells from older and male healthy individuals but reduced in asthma. Respirology 2021; 26: 442-451.

8 Eapen MS, Lu W, Hackett TL, et al. Dysregulation of endocytic machinery and ACE2 in small airways of smokers and COPD patients can augment their susceptibility to SARS-CoV-2 (COVID-19) infections. Am J Physiol Lung Cell Mol Physiol 2021; 320: L158-L163.

9 Eapen MS, Hansbro PM, McAlinden K, et al. Abnormal M1/M2 macrophage phenotype profiles in the small airway wall and lumen in smokers and chronic obstructive pulmonary disease (COPD). Sci Rep 2017; 7: 13392.

10 Saad MI, McLeod L, Hodges C, et al. ADAM17 deficiency protects against pulmonary emphysema. Am J Respir Cell Mol Biol 2021; 64: 183-195. 